\title{
Oxetane Substrates of Human Microsomal Epoxide Hydrolase ${ }^{\sqrt{\S}}$
}

\author{
Francesca Toselli, Marlene Fredenwall, Peder Svensson, Xue-Qing Li, Anders Johansson, \\ Lars Weidolf, and Martin A. Hayes
}

Cardiovascular and Metabolic Diseases, Innovative Medicines and Early Development, AstraZeneca, Mölndal, Sweden (F.T., M.F., X.-Q.L., A.J., L.W., M.A.H.); and Integrative Research Laboratories, Arvid Wallgrens Backe 20, Gothenburg, Sweden (P.S.)

Received April 24, 2017; accepted June 5, 2017

\begin{abstract}
Oxetanyl building blocks are increasingly used in drug discovery because of the improved drug-like properties they confer on drug candidates, yet little is currently known about their biotransformation. A series of oxetane-containing analogs was studied and we provide the first direct evidence of oxetane hydrolysis by human recombinant microsomal epoxide hydrolase $(\mathrm{mEH})$. Incubations with human liver fractions and hepatocytes were performed with and without inhibitors of cytochrome P450 (P450), mEH and soluble epoxide hydrolase (sEH). Reaction dependence on NADPH was investigated in subcellular fractions. A full kinetic characterization of oxetane hydrolysis is presented, in both human liver microsomes and human recombinant $\mathrm{mEH}$. In human liver fractions and hepatocytes, hydrolysis by $\mathrm{mEH}$ was the only oxetane ring-opening metabolic route, with no contribution
\end{abstract}

from sEH or from cytochrome P450-catalyzed oxidation. Minimally altering the structural elements in the immediate vicinity of the oxetane can greatly modulate the efficiency of hydrolytic ring cleavage. In particular, higher $\mathrm{p} K_{\mathrm{a}}$ in the vicinity of the oxetane and an increased distance between the oxetane ring and the benzylic nitrogen improve reaction rate, which is further enhanced by the presence of methyl groups near or on the oxetane. This work defines oxetanes as the first nonepoxide class of substrates for human $\mathrm{mEH}$, which was previously known to catalyze the hydrolytic ring opening of electrophilic and potentially toxic epoxide-containing drugs, drug metabolites, and exogenous organochemicals. These findings will be of value for the development of biologically active oxetanes and may be exploited for the biocatalytic generation of enantiomerically pure oxetanes and diols.

\section{Introduction}

Oxetane rings are becoming widely incorporated in novel drug design because of the improved properties that they confer on drug candidates (Wuitschik et al., 2010; Bull et al., 2016). The use of oxetanyl groups as alternatives to, e.g., gem-dimethyl groups leads to a significant increase in solubility and lower lipophilicity while maintaining steric bulk. The introduction of the spiro-oxetanylazetidinyl moiety has also been pursued as a means of providing favorable physicochemical and pharmacokinetic properties compared with larger ring systems (Wuitschik et al., 2010; Bull et al., 2016) and was recently reported in the discovery of the melanin-concentrating hormone receptor antagonist AZD1979 (3-(4-(2-Oxa-6-azaspiro[3.3]heptan-6-ylmethyl)phenoxy)azetidin-1-yl)(5-(4-methoxyphenyl)-1,3,4-oxadiazol-2-yl)methanone (Supplemental Fig. 1) (Johansson et al., 2016). In subsequent studies using selective inhibitors in human liver fractions, hydrolytic ring opening of the spiro-oxetanyl ring system in this compound was observed and found to be a novel reaction catalyzed by the human microsomal epoxide hydrolase ( $\mathrm{mEH}$ ) (Li et al., 2016).

$\mathrm{mEH}$ (EC 3.3.2.9) is a highly conserved drug-metabolizing enzyme and a member of the $\alpha / \beta$-hydrolase fold family of proteins (Morisseau

This work was presented in preliminary form at the 21st International Symposium on Microsomes and Drug Oxidations; 2016 Oct 2-6, Davis, CA. https://doi.org/10.1124/dmd.117.076489.

SThis article has supplemental material available at dmd.aspetjournals.org. and Hammock, 2005; Václavíková et al., 2015). mEH is widely expressed in the human body and typically catalyzes the hydrolytic ring opening of epoxide-containing exogenous organochemicals, thus playing an important role in the detoxification of electrophilic and potentially toxic drugs and drug metabolites (Morisseau and Hammock, 2005; Václavíková et al., 2015).

For the continued development of oxetane-containing drug candidates, knowledge on the enzymology of their metabolism is crucial to predict drug-drug interaction risk, model drug exposure in a variety of patient populations, and fulfill regulatory requirements. The objective of the present study was to investigate simple oxetanyl analogs of (3-(4(2-Oxa-6-azaspiro[3.3]heptan-6-ylmethyl)phenoxy)azetidin-1-yl)(5(4-methoxyphenyl)-1,3,4-oxadiazol-2-yl)methanone and to probe the influence on metabolic ring opening of different structural features in the vicinity of the oxetanyl ring. It is shown that oxetanes are exclusively ring opened via hydrolysis by human $\mathrm{mEH}$ and that several structural variations in the vicinity of the oxetane greatly affect the rate of hydrolysis. Oxetane hydrolysis by human recombinant $\mathrm{mEH}$, with full kinetic parameters, is also reported, providing the first direct evidence of this new activity of human $\mathrm{mEH}$. This work describes a new class of substrates for this important enzyme and defines hydrolysis as a major metabolic ring-opening pathway for oxetanes.

\section{Materials and Methods}

Materials. Pooled, mixed-gender human liver microsomes (HLMs) were purchased from BD Biosciences (Bedford, MA). Pooled, mixed-gender human

ABBREVIATIONS: 1-ABT, 1-aminobenzotriazole; cSO, cis-stilbene oxide; DMSO, dimethylsulfoxide; 11,12-EET, ( $( \pm) 11,12-e p o x y-5 Z, 8 Z, 14 Z-$ eicosatrienoic acid; EH, epoxide hydrolase; HLC, human liver cytosol; HLM, human liver microsome; KTZ, ketoconazole; mEH, microsomal epoxide hydrolase; MS, mass spectrometry; P450, cytochrome P450; PRG, progabide; Q-TOF, quadrupole time-of-flight; sEH, soluble epoxide hydrolase; $t$-AUCB, trans-4-[4-(1-adamantylcarbamoylamino)cyclohexyloxy]benzoic acid; UHPLC, ultra-high-performance liquid chromatography; VPD, valpromide. 
liver cytosol (HLC) and cryopreserved hepatocytes were purchased from Bioreclamation IVT (Frankfurt am Main, Germany). Recombinant human mEH was a generous gift from Dr. B. D. Hammock (University of California Davis, Davis, CA). Ketoconazole (KTZ), 1-aminobenzotriazole (1-ABT), progabide (PRG) and trans-4-[4-(1-adamantylcarbamoylamino)cyclohexyloxy]benzoic acid ( $t$-AUCB) were obtained from AstraZeneca Compound Management (AstraZeneca R\&D Gothenburg, Sweden). Valpromide (VPD), styrene oxide, cis-stilbene oxide ( $c \mathrm{SO}$ ), hydrobenzoin (the product of $c \mathrm{SO}$ ring opening), bovine serum albumin, and NADPH were purchased from Sigma Aldrich (St. Louis, MO), and $( \pm) 11,12$-Epoxy-5Z,8Z,14Z-eicosatrienoic acid (11,12-EET) was purchased from Cayman Chemicals (Ann Arbor, MI). Oxetanes 1-9 and 1520, and diols 10-14, 21, and 22 were synthesized and characterized at Medicinal Chemistry, Cardiovascular and Metabolic Diseases, Innovative Medicines and Early Development Biotech Unit, AstraZeneca (Gothenburg, Sweden), as detailed in the Supplemental Material. All other chemicals were of the highest quality commercially available.

Incubations with Human Liver Subcellular Fractions and Selective Inhibitors. The contribution of different liver enzymes to the metabolism of compounds 1-9 and 15-20 (see Tables 1 and 2 for structures) was monitored in HLMs and HLC in the presence of inhibitors. $c$ SO and 11,12-EET were used in parallel incubations as positive controls for metabolism by $\mathrm{mEH}$ and soluble epoxide hydrolase (sEH), respectively (Chacos et al., 1983; Gill et al., 1983a). The following inhibitors were used (final concentrations): KTZ [cytochrome P450 (P450) inhibitor; $200 \mu \mathrm{M}$ ], VPD (mEH inhibitor; 10-1000 $\mu \mathrm{M}$ ), or PRG (mEH inhibitor; $100 \mu \mathrm{M})$, in HLMs; and VPD $(200 \mu \mathrm{M})$ or $t$-AUCB (sEH inhibitor; $20 \mu \mathrm{M}$ ), in HLC. Concentrations of all inhibitors were chosen to be in excess of their inhibition constants reported in the literature (Kerr et al., 1989; Kroetz et al., 1993; Emoto et al., 2003; Liu et al., 2009); at the concentration used, KTZ was expected to inhibit all major liver P450s (Emoto et al., 2003).

Compounds $1-9(10 \mu \mathrm{M})$ or $c \mathrm{SO}(100 \mu \mathrm{M})$ were incubated individually with either HLMs or HLC $(1 \mathrm{mg}$ protein/ml) in sodium phosphate buffer $(0.1 \mathrm{M}, \mathrm{pH}$ $7.4,50 \mu \mathrm{l}$ ) for 1 hour at $37^{\circ} \mathrm{C}$ with constant shaking. Compounds $15-20$ were incubated in the same manner, but only with HLMs, whereas 11,12-EET $(10 \mu \mathrm{M})$ was incubated only with HLC. The subcellular fractions were preincubated in buffer with or without NADPH $(1 \mathrm{mM})$ plus inhibitor or vehicle for 3 minutes, and then reactions were started by the addition of substrate. Chemical stability of compounds 1-9 and 15-20 in buffer was also assessed in similar incubations, lacking the liver fractions and the inhibitors. Final solvent concentrations were kept constant in all reactions and were $\leq 0.3 \%(\mathrm{v} / \mathrm{v})$ acetonitrile and $\leq 1.1 \%(\mathrm{v} / \mathrm{v})$ dimethylsulfoxide (DMSO); incubations with PRG and matching controls also contained $0.5 \%(\mathrm{v} / \mathrm{v})$ methanol; incubations in HLC with 11,12-EET also contained $1.6 \%(\mathrm{v} / \mathrm{v})$ ethanol. All assays were performed in duplicate, in roundbottom 96 -well plates.

Reactions were quenched by mixing with either one $(c \mathrm{SO})$ or three $(1-9,15-$ 20 , and 11,12-EET) volumes of ice-cold acetonitrile. After centrifugation at $4000 \mathrm{~g}$ for 20 minutes, $50 \mu \mathrm{l}$ of the supernatant was mixed with an equal volume of water, and the resulting mixture was analyzed by either ultra-high-performance liquid chromatography (UHPLC)/quadrupole time-of-flight (Q-TOF) mass spectrometry (MS) ( $1-9,15-20$, and 11,12-EET] or UHPLC/UV (cSO), as described in detail subsequently.

Incubations with Human Hepatocytes. The metabolism of 1-9, 15-20, and $c \mathrm{SO}$ was also assessed in human hepatocytes, with or without inhibitors. Inhibitors used were (final concentrations): 1-ABT (P450 inhibitor; 1-2 mM), VPD (100-200 $\mu \mathrm{M})$ or $t$-AUCB (10-20 $\mu \mathrm{M})$. At the concentrations used, 1-ABT was expected to inhibit strongly all major liver P450s (Emoto et al., 2003).

Cryopreserved hepatocytes were thawed and resuspended in Leibovitz's L-15 medium to a final concentration of $10^{6}$ cells $/ \mathrm{ml}$. Cells were preincubated for 7 minutes at $37^{\circ} \mathrm{C}$ with inhibitor or vehicle under constant shaking, and reactions were then initiated by the addition of substrate $(4 \mu \mathrm{M})$. Chemical stability of substrates in Leibovitz's L-15 medium was assessed in similar incubations, lacking hepatocytes and inhibitors. Final solvent concentrations were $0.04 \%$ DMSO and $0.08 \%$ acetonitrile in all reactions, and incubations $(50 \mu \mathrm{l})$ were performed in duplicate, in flat-bottom 96-well plates. Reactions were quenched after 2 hours and processed as described previously for incubations with subcellular fractions.

Determination of Kinetic Constants for Oxetane Hydrolysis. Human recombinant $\mathrm{mEH}$ and HLMs were used to examine the kinetics of oxetane hydrolysis. Conditions for linear product formation with respect to time and

TABLE 1

Physicochemical properties of compounds 1-9 and percentage of dihydrodiol metabolites formed in incubations with HLMs

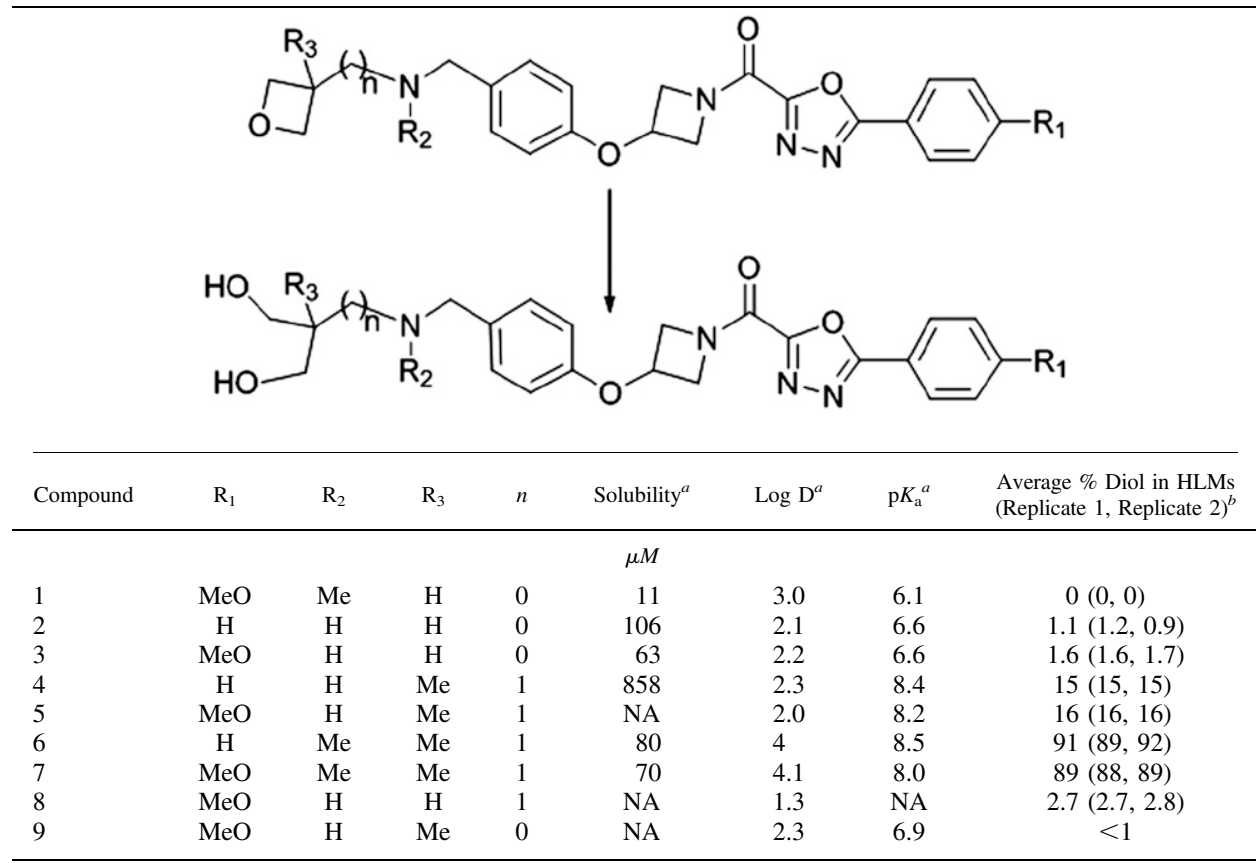

NA, not available.

${ }^{a}$ Solubility, $\log \mathrm{D}_{7.4}$ HPLC and $\mathrm{p} K_{\mathrm{a}}$ were measured as described previously (Boström et al., 2012).

${ }^{b}$ Diol levels were determined from duplicate incubations (60 minutes) of $10 \mu \mathrm{M}$ substrate with HLMs, without NADPH. Diol metabolite levels are expressed as a percentage of the sum of parent + diol peak areas. 
TABLE 2

Physicochemical properties of compounds 15-20 and percentage of dihydrodiol metabolites formed in incubations with HLMs

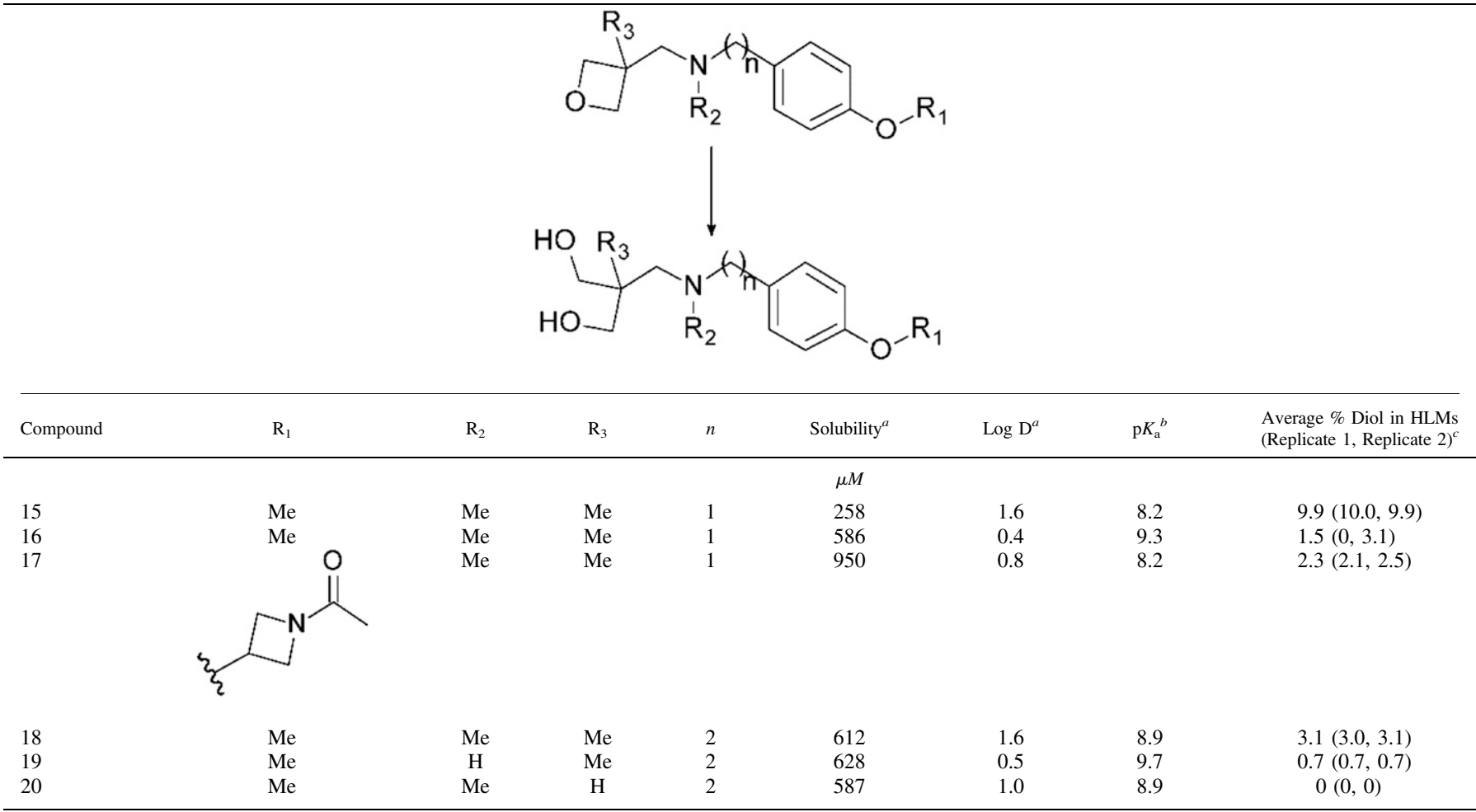

${ }^{a}$ Solubility and $\log \mathrm{D}_{7.4}$ HPLC were measured as described previously (Boström et al., 2012).

${ }^{b}$ Calculated values.

${ }^{c}$ Diol levels were determined from duplicate incubations (60 minutes) of $10 \mu \mathrm{M}$ substrate with HLMs, without NADPH. Diol metabolite levels are expressed as a percentage of the sum of parent + diol peak areas.

protein concentration were assessed with the lowest substrate concentration later used in the substrate-saturation curves. No or negligible hydrolysis of oxetanes in compounds $1-3,8$, and 9 was observed in these preliminary assays, thus further kinetic experiments were only performed with compounds 4-7. From the second set of compounds 15 and 18 were selected for full kinetic characterization.

In assays with the recombinant enzyme, substrates (5-200 $\mu \mathrm{M}$ for compounds 5-7; 5-300 $\mu \mathrm{M}$ for compound 4 ; or 10-200 $\mu \mathrm{M}$ for compounds 15 and 18) were incubated with $1.9 \mu \mathrm{g} / \mathrm{ml}$ (compounds $4-7$ ) or $5.6 \mu \mathrm{g} / \mathrm{ml}$ (compounds 15 and 18) of recombinant $\mathrm{mEH}$, for either 10 minutes (compound 6), 30 minutes (compounds 5 and 7), 40 minutes (compound 4), or 60 minutes (compounds 15 and 18). The recombinant enzyme was thawed and diluted in cold Tris- $\mathrm{HCl}$ buffer $(0.1 \mathrm{M}, \mathrm{pH} 9.0)$ supplemented with $0.1 \mathrm{mg} / \mathrm{ml}$ bovine serum albumin, which is necessary to stabilize the purified protein and help solubilize lipophilic substrates (Morisseau and Hammock, 2007). This mixture was preincubated at $37^{\circ} \mathrm{C}$ with constant shaking for 5 minutes, before starting the reactions with the addition of substrate (100 $\mu$ l final reaction volume). An identical volume of substrate solution was added to each reaction directly from high-concentration acetonitrile stocks (compounds 4-7) or acetonitrile/DMSO stocks (compounds 15 and 18), to maintain the final solvent concentration $\leq 3 \%(\mathrm{v} / \mathrm{v})$ in all incubations. $\mathrm{mEH}$ activity has been reported to be unaffected by acetonitrile or DMSO concentrations up to 3\% (v/v) (Seidegård and DePierre, 1980; Müller et al., 1997). Reactions were quenched and processed as described previously. All assays were performed in duplicate (compounds 4, 15, and 18) or triplicate (all other substrates), in round-bottom 96-well plates. To build calibration curves, synthetic diol standards $10-13,21$, and $22(0.01-2.5 \mu \mathrm{M})$ were incubated, processed, and analyzed in the same manner as the substrates, except without enzyme.

Kinetic experiments with HLMs were performed similarly, but with the following exceptions: substrates (10-200 $\mu \mathrm{M}$ for compounds 5-7, 15, and 18 or $10-300 \mu \mathrm{M}$ for compound 4) were incubated with $5 \mu \mathrm{g} / \mathrm{ml}$ (compounds 4-7) or $15 \mu \mathrm{g} / \mathrm{ml}$ (compounds 15 and 18) of HLM protein for 40 minutes (compounds 4 7) or 60 minutes (compounds 15 and 18), in sodium phosphate buffer ( $0.1 \mathrm{M}, \mathrm{pH}$
7.4). Assays were performed in duplicate (compounds 7, 15, and 18) or triplicate (all other substrates) and the concentration range for calibration curves with synthetic diol standards was 0.1-5 $\mu \mathrm{M}$. Under the conditions used, with no other metabolite formed than the ring-opened diol, substrate depletion did not exceed $6 \%$ with either enzyme system.

Determination of $\mathrm{IC}_{50}$ for Inhibition of $c$ SO Hydrolysis by VPD and Compounds 1-9. Human recombinant $\mathrm{mEH}$ was diluted to $1.9 \mu \mathrm{g} / \mathrm{ml}$ in Tris$\mathrm{HCl}$ buffer $(0.1 \mathrm{M}, \mathrm{pH} 9.0)$ containing $0.1 \mathrm{mg} / \mathrm{ml}$ bovine serum albumin. Compounds $1-9(0-200 \mu \mathrm{M})$ or VPD $(0-2 \mathrm{mM})$ were added from acetonitrile stocks and the mixture was preincubated for 5 minutes at $37^{\circ} \mathrm{C}$ with constant shaking. Then, $c \mathrm{SO}$ was added from a DMSO stock to a final concentration equal to its $K_{\mathrm{m}}$ value (140 $\mu \mathrm{M}$ with the human recombinant enzyme) (Morisseau et al., 2011) and incubated for a further 10 minutes. Preliminary experiments confirmed a linear hydrobenzoin product formation in these conditions (data not shown). Final solvent concentrations were $0.56 \%$ DMSO and $2 \%$ acetonitrile in all incubations (100 $\mu$ l total reaction volume). All assays were performed in triplicate in round-bottom 96-well plates. Generation of calibration curves and processing of the reactions were done as described in the previous section.

Metabolite Profiling and Data Analysis for Selective Inhibition Assays. For incubations with 1-9, 15-20, or 11,12-EET, UHPLC/Q-TOF analysis of metabolites from selective inhibition assays was performed as described previously ( $\mathrm{Li}$ et al., 2016), with the following modifications: a different gradient profile was used with the initial mobile phase (90:10 A:B) transitioning to 70:30 A:B over 6 minutes and the mass spectrometer was a Synapt G2 Q-TOF (Waters (Milford, MA)). Mobile phase A was $0.1 \%$ formic acid in water (for reactions with oxetanes or 11,12-EET) or water (for reactions with $c \mathrm{SO}$ ) and mobile phase B was acetonitrile. An $\mathrm{MS}^{\mathrm{E}}$ method with two separate scan functions programmed with independent collision energies was used for data acquisition. Trap collision energy in function 1 was $4 \mathrm{~V}$ and in function 2 an energy ramp of 15-35 V was used; the transfer cell collision energy was $20 \mathrm{~V}$. The entire system was operated using MassLynx (Waters, version 4.1), and data were processed with MetaboLynx version 4.1. $\mathrm{MS}$ and $\mathrm{MS}^{\mathrm{E}}$ spectra were compared between the parent 
compound and metabolites to identify metabolite structures and site(s) of modification in the substrate molecule. UHPLC/Q-TOF MS ${ }^{\mathrm{E}}$ characteristics of compounds 1-9 and their hydrated metabolites are listed in Supplemental Table 1, although no $\mathrm{MS}^{\mathrm{E}}$ spectra could be obtained for compounds 15-20. Products from reactions with $c \mathrm{SO}$ were analyzed by UV detection. The UHPLC eluate was introduced into an Acquity UV-PDA detector (Waters), and the diol hydrobenzoin metabolite in the experimental samples was identified by comparing the retention time with that of the authentic standard. Retention times for hydrobenzoin and $c \mathrm{SO}$ were 3.27 and 5.38 minutes, respectively. Metabolite peaks on the trace from the full UV scan (210-400 nm) were integrated using the TargetLynx tool (Waters).

Peak areas were used for the relative quantification of diol metabolites in HLM incubations without NADPH (reported in Table 1), by calculating

$$
\text { Relative diol } \%=\frac{\text { Diol }_{\text {area }}}{\text { Diol }_{\text {area }}+\text { Parent }_{\text {area }}} \times 100
$$

For incubations with inhibitors (or those lacking NADPH), relative diol levels were expressed as a percentage of the corresponding peak areas in control incubations with vehicle and NADPH.

Metabolite Quantification and Data Analysis for Kinetic Assays. Chromatographic separation of metabolites from kinetic assays was performed on a Waters Acquity UHPLC HSS T3 column $(2.1 \times 100 \mathrm{~mm}, 1.8 \mu \mathrm{m}$ for compounds 4-7) or a Waters Acquity UHPLC BEH column $(2.1 \times 100 \mathrm{~mm}$, $1.7 \mu \mathrm{m}$ for compounds 15 and 18) operated by an Acquity UHPLC system. To improve the separation between diols and parent compounds, mobile phase A was changed from $0.1 \%$ aqueous formic acid to $10 \mathrm{mM}$ ammonium acetate in water; mobile phase B was acetonitrile. The initial mobile phase was 90:10 A:B, transitioning to $10: 90 \mathrm{~A}: \mathrm{B}$ over 6 minutes using a linear gradient, with a 7.7minute total run time. The flow rate was $0.5 \mathrm{ml} / \mathrm{min}$ and the column oven was set to $45^{\circ} \mathrm{C}$. A 1-minute window of UHPLC eluate, centered on the retention time of each diol metabolite, was introduced into a Xevo G2-S Q-TOF mass spectrometer for compounds 4-7 (Waters) or a Synapt G2 Q-TOF for compounds 15 and 18 (Waters), operating as described previously. Diols formed in incubations with compounds $4-8,15$, and 18 were quantified by external calibration as described in the previous sections, using TargetLynx (Waters). The extracted ion chromatograms corresponding to the protonated molecular ion of the diol were generated with a mass tolerance of $30 \mathrm{mDa}$.
To determine the $K_{\mathrm{m}}$ and $V_{\max }$ values, initial velocities were plotted versus the corresponding substrate concentration and fitted to the Michaelis-Menten equation by nonlinear regression, using GraphPad Prism version 6.07 for Windows (GraphPad Software, La Jolla, CA). Detection and quantification of hydrobenzoin formed in $\mathrm{IC}_{50}$ assays with $c \mathrm{SO}$ was performed by UHPLC/UV as described in the previous section, except that $10 \mathrm{mM}$ ammonium acetate was used as mobile phase A instead of water to avoid coelution of hydrobenzoin with oxetanes or diols. Velocities of hydrobenzoin formation were plotted against the logarithm of the corresponding inhibitor concentration and fitted to the one-site competitive binding equation by nonlinear regression, using GraphPad Prism.

Calculations of Ring-Strain Energies and Determination of $\mathrm{p} K_{\mathrm{a}}$. A detailed account of ring-strain energy calculations for relevant truncated fragments of compounds 1-9 is given in the Supplemental Material. Determination of solubility and $\log \mathrm{D}$ for all oxetanes, and $\mathrm{p} K_{\mathrm{a}}$ values for compounds 1-9 was performed as described previously (Boström et al., 2012).

\section{Results}

Oxetanes Are Opened Exclusively via Hydrolysis by mEH. Compounds 1-9, 15-20, cSO, and 11,12-EET were incubated with human liver subcellular fractions or hepatocytes in the presence or absence of inhibitors of P450s, mEH, and sEH. Ring opening of the substrates in incubations with liver fractions was also monitored with or without NADPH. All oxetanes were metabolized to a variety of products in the presence of NADPH (see Fig. 1A for an example with compound 5 incubated with HLMs). Metabolites included products with a mass increase of $18 \mathrm{Da}\left(\mathrm{H}_{2} \mathrm{O}\right)$ with respect to substrate and its $\mathrm{N}$ - or $O$-demethylation products.

The oxetanylmethylaminyl moiety was the only part of each compound not retained in any of the fragment ions detected under the $\mathrm{MS}^{\mathrm{E}}$ conditions used, and no fragment of the hydrated metabolite showed a mass shift of +18 Da relative to that of the parent (see representative MS and $\mathrm{MS}^{\mathrm{E}}$ spectra for compound 5 in Fig. 2 and MS and $\mathrm{MS}^{\mathrm{E}}$ data for all compounds in Supplemental Table 1). The hydrated metabolites formed from each parent compound were then found to coelute with the corresponding synthesized diols (see Fig. 1B for

A

A Compound 5

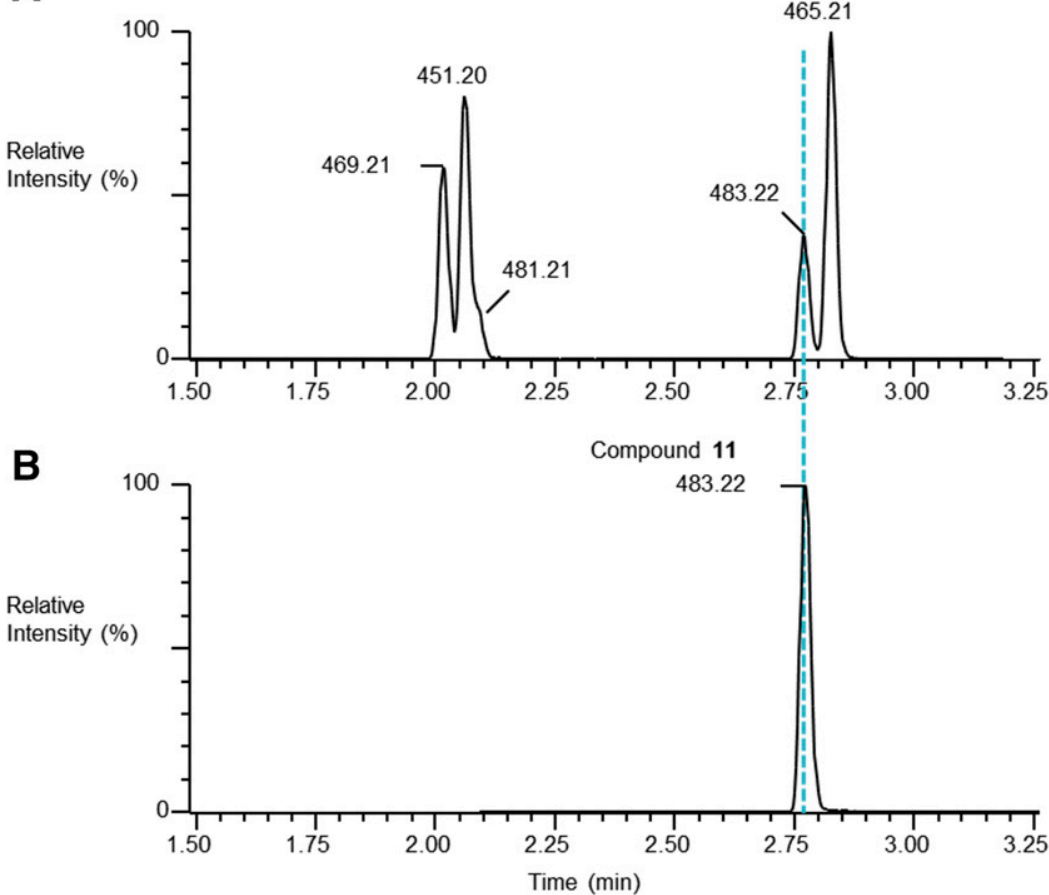

Fig. 1. Metabolism of compound 5. UHPLC-MS extracted ion chromatograms are shown for (A) compound 5 and its metabolites formed after 60-minute incubation with HLMs and NADPH and (B) synthetic diol 11. Peak labels are $\mathrm{m} / \mathrm{z}$ values and correspond to parent $(\mathrm{m} / \mathrm{z} 465.21)$, hydrolysis $(\mathrm{m} / \mathrm{z} 483.22)$, oxidation $(\mathrm{m} / \mathrm{z}$ 481.21), demethylation $(\mathrm{m} / \mathrm{z} 451.20)$, and hydrolysis plus demethylation $(\mathrm{m} / \mathrm{z}, 469.21)$. 
representative chromatograms for compound 5 and Supplemental Fig. 5 for the synthetic diol structures), and $\mathrm{MS}^{\mathrm{E}}$ spectra of each metabolite/ synthetic diol pair were identical (Fig. 2).

Hydration (addition of the elements of $\mathrm{H}_{2} \mathrm{O}$ ) for all test compounds was NADPH independent in incubations with HLMs (see Fig. 3 for an example with compound 6 and Supplemental Fig. 2 for all other compounds). In contrast, the formation of diols from all oxetanes and the $\mathrm{mEH}$ probe substrate $c \mathrm{SO}$, but not from the $\mathrm{sEH}$ probe substrate 11,12EET, was essentially abolished in the presence of high concentrations of the $\mathrm{mEH}$ inhibitor VPD in liver fractions and hepatocytes (Fig. 3; Supplemental Fig. 2). Similar results were obtained with the structurally unrelated $\mathrm{mEH}$ inhibitor PRG (data not shown). High concentrations of the P450 inhibitors KTZ and 1-ABT did not inhibit hydration of any compound (Fig. 3; Supplemental Fig. 2), while they greatly decreased demethylation of compounds 1, 3, 5-7, 9, and 15-20 (data not shown), indicating that a functional P450 system was present and could be effectively inhibited in the assay conditions used. Some degree of inhibition of diol formation by $\mathrm{P} 450$ inhibitors was observed for a compounds 4 and 15-17 in HLMs and 2, 5, 8, 16, and 17 in hepatocytes (Supplemental Fig. 2), but this was also observed for hydrolysis of the mEH probe substrate $c \mathrm{SO}$ (Supplemental Fig. 2). In addition, incubation of these and the other oxetanes with both NADPH and VPD completely abolished diol formation (Fig. 3; Supplemental Fig. 2). These data suggested that the apparent reduction of oxetane hydrolysis by P450 inhibitors for these compounds was likely due to experimental variation between samples, rather than a true contribution of P450s to oxetane ring opening. Conversely, diol formation from other compounds (1-3, 6, 7, and 15-18 in HLMs and 3, 4, 6, 7, and 9 in hepatocytes) increased in the absence of a functional P450 system (i.e., either in incubations without $\mathrm{NADPH}$ or in the presence of KTZ or 1-ABT) (Fig. 3; Supplemental
Fig. 2), while formation of the demethylated metabolites increased when mEH was inhibited (data not shown). Thus, inhibition of P450, or lack of $\mathrm{NADPH}$, increased the availability of substrate for $\mathrm{mEH}$-mediated metabolism, and conversely, inhibition of $\mathrm{mEH}$ increased substrate availability for P450-mediated metabolism. This indicated an apparent competition between $\mathrm{mEH}$ and $\mathrm{P} 450$ s for the substrate and a lack of nonspecific inhibition of $\mathrm{P} 450$ activity by $\mathrm{mEH}$ inhibitors. Coincubation with the sEH inhibitor $t$-AUCB failed to inhibit hydration of oxetanes and $\mathrm{cSO}$ in hepatocytes (Fig. 3; Supplemental Fig. 2), whereas it abolished hydrolysis of the sEH probe substrate 11,12-EET in HLC (Supplemental Fig. 2). The same pattern of inhibition of oxetane hydration seen in HLMs and hepatocytes by VPD was also observed in HLC, where minor NADPH-independent diol formation occurred (Fig. 3; Supplemental Fig. 2). This is likely due to low levels of $\mathrm{mEH}$ contamination in the cytosol, as previously reported (Gill et al., 1982, 1983b). No diol formation was observed when the enzyme source was omitted (Fig. 3; Supplemental Fig. 2). The inhibitory profile seen with the selective epoxide hydrolase (EH) inhibitors VPD and $t$-AUCB against hydrolysis of the probe substrates $c \mathrm{SO}$ and 11,12-EET indicated that the assay was specific and effective at differentiating between metabolism catalyzed by the two forms of human EH.

Specific Structural Elements Modulate Oxetane Hydrolysis in HLMs and Hepatocytes. The oxetane substrates were hydrolyzed to varying degrees by $\mathrm{mEH}$ (Tables 1 and 2). Diol formation was absent or minor for compounds $1-3,9$, and 15-20, but was a major metabolic route for compounds 4-8. Among the full-length analogs, poor (1-3 and 9) and good (4-8) oxetane substrates also had significantly different $\mathrm{p} K_{\mathrm{a}}$ values $(6.5 \pm 0.2$ and $8.2 \pm 0.1$, respectively; $P<$ 0.001 , two-tailed unpaired $\mathrm{T}$ test) (Table 1), although they had similar solubilities, lipophilicities (Table 1), and theoretical ring-strain energies
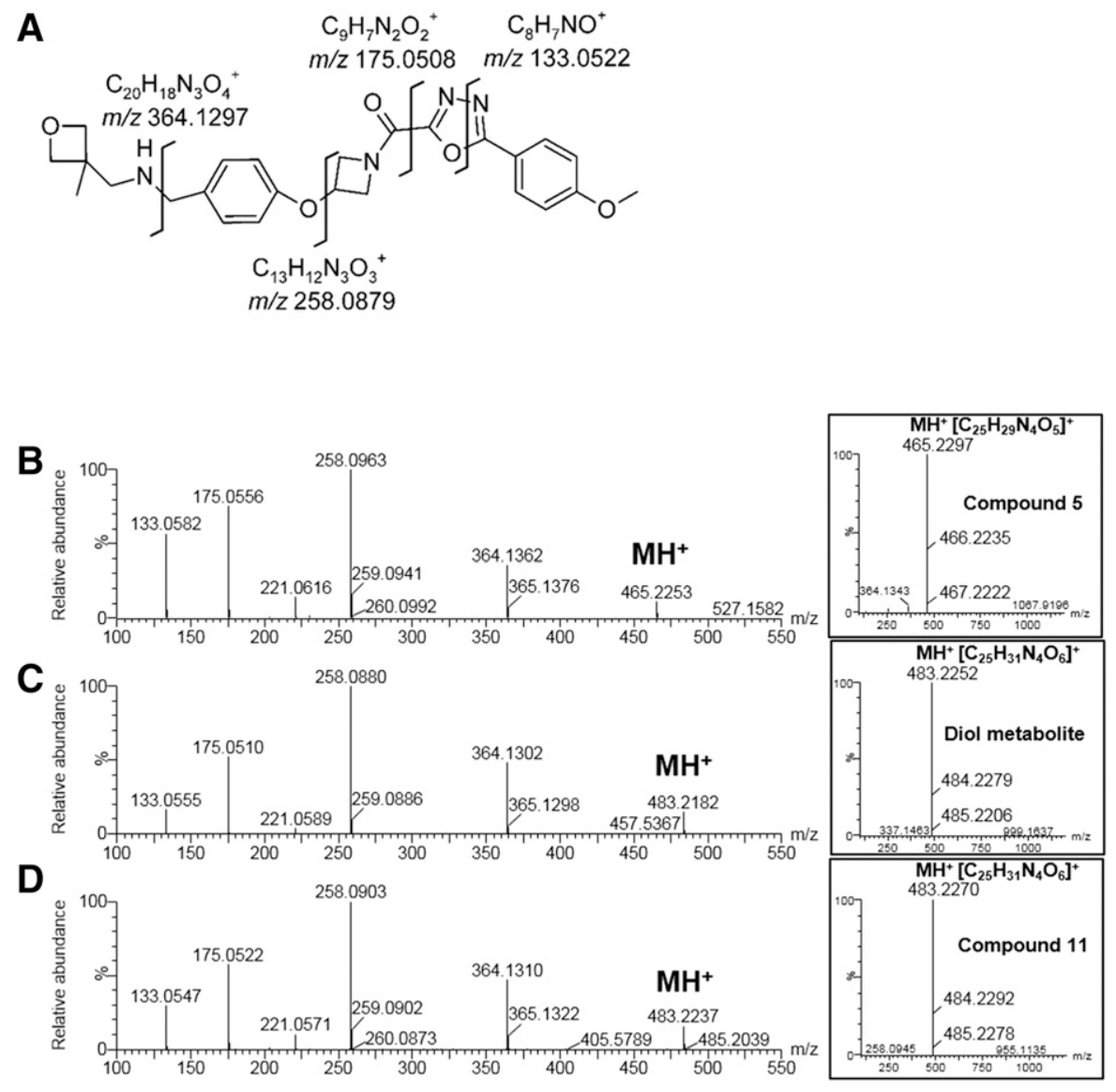

Fig. 2. Tentative fragmentation pattern (A) and $M S^{\mathrm{E}}$ spectra of compound 5 (B), its diol metabolite formed in incubations with HLMs (C) and the synthesized diol 11 (D). Insets are full-scan MS spectra. 

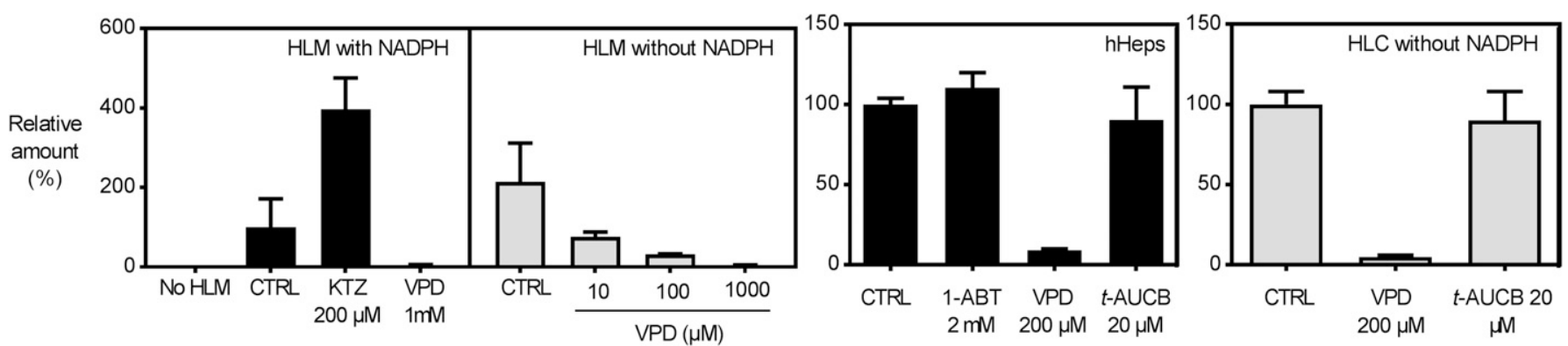

Fig. 3. Effect of P450 inhibitors KTZ and 1-ABT, the mEH inhibitor VPD and the sEH inhibitor $t$-AUCB $(20 \mu \mathrm{M})$ on the hydration of compound 6 for HLMs, human hepatocytes (hHeps), and HLC. Assay conditions were: $10 \mu \mathrm{M}$ substrate, 60-minute incubation with $1 \mathrm{mg} / \mathrm{ml} \mathrm{HLM}$ or HLC protein; and $4 \mu \mathrm{M}$ substrate, 2-hour incubation with $10^{6}$ hepatocytes $/ \mathrm{ml}$. Results are expressed as a \% of the diol formed in reactions with NADPH and no inhibitor (CTRL), and bars are averages of duplicate measurements + range. Similar results were also obtained with the $\mathrm{mEH}$ inhibitor progabide.

(Supplemental Table 2). However, this trend in $\mathrm{p} K_{\mathrm{a}}$ was not observed for the chain-shortened analogs (15-20), which showed broadly similar calculated $\mathrm{p} K_{\mathrm{a}}$ values (Table 2 ).

Human Recombinant mEH Hydrolyses Oxetanes with the Same Substrate Preference as HLMs. To more fully understand the interaction of $\mathrm{mEH}$ with oxetanes, kinetic parameters for the hydrolysis of each of the full-length analogs (1-9) and two of the chain-shortened analogs (15 and 18) were determined in separate incubations with human recombinant $\mathrm{mEH}$ or HLMs. Among full-length compounds 1-9, both enzyme systems showed the same substrate preference and only efficiently hydrolyzed compounds 4-7. All four compounds 4-7 exhibited MichaelisMenten-type kinetics with both the recombinant enzyme and HLM and showed similar $K_{\mathrm{m}}$ values (substrate-saturation curves are shown in Supplemental Figs. 3 and 4; Table 3). Hydrolysis of compounds 15 and 18 was less efficient and meant that $K_{\mathrm{m}}$ and $V_{\mathrm{max}}$ could only be determined with HLMs since within the substrate concentration range chosen the velocity remained linear with the recombinant enzyme (Supplemental Figs. 3 and 4; Table 3). No inhibition of $c \mathrm{SO}$ metabolism by compounds 1-9 was observed with recombinant $\mathrm{mEH}$ when using a substrate concentration corresponding to its $K_{\mathrm{m}}$ (data not shown). VPD, when tested under the same conditions, inhibited $c \mathrm{SO}$ hydrolysis with an $\mathrm{IC}_{50}$ value of $7.1 \pm 0.3 \mu \mathrm{M}$.

\section{Discussion}

Oxetanes are being increasingly incorporated into the design of novel biologically active molecules (Wuitschik et al., 2010; Bull et al., 2016), yet there are few detailed reports on their biotransformation. Here, we have shown that the oxetane moiety in a series of analogs can be consistently metabolized to a diol metabolite via hydrolysis by $\mathrm{mEH}$, without contributions by any other enzyme system, and that this pathway may represent a major metabolic route depending on the structural context in which the oxetane is embedded. Oxetane rings thus represent a new class of substrates for human $\mathrm{mEH}$.

An earlier study by Stepan et al. (2011) was the first to report metabolic ring cleavage of oxetanyl moieties in HLMs, but it was unclear which enzyme specifically catalyzed the formation of diol metabolites. A more recent study described an example of an oxetanecontaining drug, EPZ015666, where the oxetanyl moiety was directly attached to a bicyclic aromatic core (Rioux et al., 2016). This compound was found to undergo ring opening to diol via P450-mediated oxidation, likely via $\alpha$-hydroxylation, ring opening to the aldehyde, and finally reduction to diol (Rioux et al., 2016). This is in contrast to our recent (Li et al., 2016) and current findings and suggests that the environment of the oxetanyl moiety is a key to determining the metabolic fate of this class of compounds.

In the series of analogs analyzed in this study, altering the structural elements in the vicinity of the oxetane greatly affected the efficiency of hydrolytic ring cleavage by $\mathrm{mEH}$. Seven compounds were studied initially (1-7), of which four (4-7) showed more than $10 \%$ hydrolysis. While these data are semiquantitative, not taking the potentially different MS response between the diols and corresponding parent into account, they served the purpose of dividing the compounds into two groups:

TABLE 3

Kinetic parameters for dihydrodiol formation in reactions with compounds 4-7, 15, and $18^{a}$

\begin{tabular}{|c|c|c|c|c|c|c|}
\hline \multirow{2}{*}{ Compound } & \multicolumn{3}{|c|}{ Human Recombinant $\mathrm{mEH}$} & \multicolumn{3}{|c|}{ HLM without NADPH } \\
\hline & $K_{\mathrm{m}}$ & $V_{\max }$ & $V_{\max } / K_{\mathrm{m}}$ & $K_{\mathrm{m}}$ & $V_{\max }$ & $V_{\max } / K_{\mathrm{m}}$ \\
\hline & $\mu M$ & $\mathrm{nmol} / \mathrm{min} / \mathrm{mg}$ & & $\mu M$ & $\mathrm{nmol} / \mathrm{min} / \mathrm{mg}$ & \\
\hline 4 & $67 \pm 10$ & $17 \pm 1$ & 0.26 & $27 \pm 5$ & $1.7 \pm 0.1$ & 0.06 \\
\hline 5 & $47(43,59)$ & $17(17,17)$ & 0.36 & $26 \pm 6$ & $1.7 \pm 0.1$ & 0.07 \\
\hline 6 & $89 \pm 14$ & $22 \pm 2$ & 0.24 & $28 \pm 8$ & $6.6 \pm 0.7$ & 0.27 \\
\hline 7 & $50 \pm 7$ & $35 \pm 2$ & 0.69 & $51(48,56)$ & $16(15,16)$ & 0.31 \\
\hline 15 & $\bar{b}$ & $b$ & $b$ & $352(239,695)$ & $0.9(0.8,1.4)$ & 0.003 \\
\hline 18 & $684(650,722)$ & $2.8(2.7,3.0)$ & 0.004 & $139(119,165)$ & $0.4(0.3,0.4)$ & 0.003 \\
\hline$c \mathrm{SO}^{c}$ & 141 & 570 & 4 & $7-25$ & $17-52$ & $2.1-2.5$ \\
\hline $\mathrm{SO}^{d}$ & $e$ & $e$ & $e$ & $40-300$ & $10-25$ & $0.05-0.4$ \\
\hline
\end{tabular}

SO, styrene oxide.

${ }^{a}$ Values are the mean of triplicates \pm S.E., except for compounds 5, 15, and 18 incubated with recombinant $\mathrm{mEH}$ and compounds 7, 15, and 18 incubated with HLMs, for which the duplicate averages (replicate 1, replicate 2) are reported. Product formation was linear with respect to time and enzyme concentration.

${ }^{b}$ Kinetic parameters could not be estimated since the velocity remained linear within the substrate concentration range chosen.

${ }^{c}$ Values are from Kitteringham et al. (1996), Hosagrahara et al. (2004), and Morisseau et al. (2011).

${ }^{d}$ Values are from Seidegård and DePierre (1980), Pacifici et al. (1986), and Eugster et al. (1991).

${ }^{e}$ Not tested 
poor (1-3) and good (4-7) $\mathrm{mEH}$ substrates. This grouping was later supported when the compounds were tested with recombinant $\mathrm{mEH}$. Two structural elements were present only in the compounds (4-7) most prone to hydrolysis, but were lacking from the others: 3-methyl substitution on the oxetane ring and a methylene group between the oxetane ring and the benzylic nitrogen. Two additional analogs were then synthesized, each containing only one of these structural elements (compounds 8 and 9). Addition of the 3-methyl substitution on the oxetane ring (compound 9) led to traces of diol formation, whereas introduction of a methylene spacer between the oxetane and the basic nitrogen (compound 8) led to an almost 2-fold increase in diol formed from the similarly substituted oxetane 3 . This indicated that hydrolysis could be improved by the presence of a methylene spacer (compound 8 versus 3). Methyl substitutions alone, whether on oxetane (9) or benzylic nitrogen (1), do not affect hydrolysis, but in combination with the methylene spacer (4-7) each methyl substitution leads to a 5-fold increase in hydrolysis. To build on these initial structure-metabolism observations and to confirm that the specific enzymology of oxetane hydrolysis was retained with a minimal oxetane-containing substrate, a set of chain-shortened analogs was synthesized with masses ranging from 221 to $318 \mathrm{Da}$ (15-20) (as outlined in the Supplemental Material). Specifically, the new set of compounds were analogs of compounds 4-8, where the 4-methoxyphenyl-oxadiazole amide-linked azetidine moiety had been replaced with a methyl group on the central phenyl ring. One analog (17) was prepared in which the 4-methoxyphenyl-oxadiazole was replaced with an acetoxy substitution on the central azetidine.

While hydrolysis was less efficient than that of the full-length analogs, most likely due to the lower lipophilicities of compounds 15-20, the chain-shortened analogs were still exclusively hydrolyzed by $\mathrm{mEH}$, with no contributions from either P450s or $\mathrm{SEH}$. A higher rate of hydrolysis of compounds 15-20 was observed with increasing methylation, as observed with the full-length analogs, whereas the presence of a methylene spacer between the amine and the phenyl ring decreased it.

When physicochemical parameters were considered as potential predictors of hydrolysis rate, it was initially observed that among fulllength analogs 1-9, good $\mathrm{mEH}$ substrates (4-8) had significantly higher $\mathrm{p} K_{\mathrm{a}}$ values; however, this did not apply to the set of chain-shortened compounds 15-20, which had calculated $\mathrm{p} K_{\mathrm{a}}$ values similar to those of compounds 4-8 despite being hydrolyzed at a considerably lower rate. Similarly, no trends in solubility, lipophilicity, or calculated ring-strain energy was observed between good and poor substrates, indicating that hydrolysis efficiencies are likely determined by active site binding properties.

Importantly, the current work presents the first direct evidence of $\mathrm{mEH}$-catalyzed oxetane hydrolysis with recombinant human $\mathrm{mEH}$, which hydrolyzed oxetanes with the same substrate preference as human liver $\mathrm{mEH}$. Kinetic experiments showed some variation in clearance $\left(V_{\max } / K_{\mathrm{m}}\right)$ between substrates, and when compared with that of $\mathrm{mEH}$ probe substrates, oxetane clearance was lower than that for $c \mathrm{SO}$, but similar to, or higher than that of styrene oxide (for reactions with HLMs) (Seidegård and DePierre, 1980; Pacifici et al., 1986; Eugster et al., 1991). Low $K_{\mathrm{m}}$ values and reaction rates are common for EHs, with turnover numbers for $\mathrm{mEH}$ substrates usually being lower than 1 second $^{-1}$ (Oesch et al., 2000). This can be explained by the reported two-step catalytic mechanism for EHs: 1) the substrate rapidly binds to the active site forming a Michaelis complex, which then reversibly alkylates the enzyme forming an ester intermediate, and 2) this intermediate then undergoes hydrolysis to form the corresponding diol (Armstrong, 1999); for a schematic illustrating reaction of a spirooxetane substrate with $\mathrm{mEH}$, see Li et al. (2016). The alkylation of the enzyme proceeds at a much faster rate $(\sim 3$ orders of magnitude faster) than the hydrolysis of the ester bond (Tzeng et al., 1996), resulting in an accumulation of the ester intermediate and a low rate of product formation (Oesch et al., 2000); therefore, low $K_{\mathrm{m}}$ values have been suggested to reflect a high degree of enzyme alkylation rather than a high affinity for the enzyme active site (Tzeng et al., 1996). Low rates of product formation thus do not necessarily reflect low efficiency of substrate consumption since the enzyme may bind more substrate than it forms diol (Oesch et al., 2000). Additionally, where $\mathrm{mEH}$ is highly expressed, e.g., in human liver (Thomas et al., 1982; de Waziers et al., 1990; Song et al., 2015), its concentration may exceed that of the substrate and thus substrate depletion in vivo may be more efficient than what is suggested by in vitro kinetic profiles. Nonetheless, the kinetic parameters evaluated according to the Michaelis-Menten model provide a means to compare the test compounds and their propensity to undergo hydrolysis by $\mathrm{mEH}$. The name of the studied enzyme implies that only epoxides are accepted substrates and therefore our findings on the broader substrate specificity should motivate a change in enzyme description (EC 3.3.2.9) to include additional small strained ring systems, i.e., oxetanes.

In conclusion, we describe the enzyme-catalyzed hydrolysis of a series of simple oxetanes by $\mathrm{mEH}$. The structural requirements for these oxetanyl-containing compounds to be accepted as substrates and hydrolyzed by $\mathrm{mEH}$ efficiently were characterized and the contributions from other enzymes were evaluated. These findings will be of value to medicinal chemists designing new oxetane-containing drug candidates. For example, it is not unusual that the metabolic clearance of an investigational drug is highly dependent on, e.g., CYP3A4, increasing the risk of drug-drug interactions with comedications. Introducing an oxetane into a chemistry series may be used as a tool to direct metabolism toward $\mathrm{mEH}$ and decrease the dependence on $\mathrm{P} 450$ metabolism. The degree to which this clearance route is tuned in or out can be adjusted using the structural modifications described herein.

This work describes the first stable, nonreactive class of substrates for human $\mathrm{mEH}$, which will allow the reliable measurement of $\mathrm{mEH}$ enzyme activity in humans and the scaling from preclinical animal species to man. Finally, one can also envision exploitation of $\mathrm{mEH}$ as a biocatalyst for racemic oxetane resolution, in a similar manner to the use of, e.g., microbial and fungal EHs with epoxides (Hechtberger et al., 1993; Steinreiber and Faber, 2001; Widersten et al., 2010). The structure-metabolism relationship between strained ring-containing drug candidates and $\mathrm{mEH}$ is being further explored in ongoing studies on compounds with diverse scaffolds.

\section{Acknowledgments}

The authors thank Dr. Bruce Hammock and Dr. Christophe Morisseau (University of California Davis, Davis, CA) for the generous gift of the recombinant enzyme, Dr. Sten Nilsson-Lill (AstraZeneca) for helpful discussions on computational methods, and Dr. Neal Castagnoli Jr. (Virginia Tech, Blacksburg, VA) and Dr. Erick Carreira (ETH Zurich, Zürich, Switzerland) for helpful discussions and critical reading of the manuscript.

\section{Authorship Contributions}

Participated in research design: Toselli, Li, Johansson, Weidolf, Hayes. Conducted experiments: Toselli, Fredenwall.

Contributed new reagents or analytic tools: Svensson.

Performed data analysis: Toselli, Fredenwall, Svensson, Li, Johansson, Weidolf, Hayes.

Wrote or contributed to the writing of the manuscript: Toselli, Fredenwall, Svensson, Li, Johansson, Weidolf, Hayes.

\section{References}

Armstrong RN (1999) Kinetic and chemical mechanism of epoxide hydrolase. Drug Metab Rev 31: 71-86.

Boström J, Hogner A, Llinàs A, Wellner E, and Plowright AT (2012) Oxadiazoles in medicinal chemistry. J Med Chem 55:1817-1830.

Bull JA, Croft RA, Davis OA, Doran R, and Morgan KF (2016) Oxetanes: recent advances in synthesis, reactivity, and medicinal chemistry. Chem Rev 116:12150-12233. 
Chacos N, Capdevila J, Falck JR, Manna S, Martin-Wixtrom C, Gill SS, Hammock BD, and Estabrook RW (1983) The reaction of arachidonic acid epoxides (epoxyeicosatrienoic acids) with a cytosolic epoxide hydrolase. Arch Biochem Biophys 223:639-648.

de Waziers I, Cugnenc PH, Yang CS, Leroux JP, and Beaune PH (1990) Cytochrome P 450 isoenzymes, epoxide hydrolase and glutathione transferases in rat and human hepatic and extrahepatic tissues. J Pharmacol Exp Ther 253:387-394.

Emoto C, Murase S, Sawada Y, Jones BC, and Iwasaki K (2003) In vitro inhibitory effect of 1-aminobenzotriazole on drug oxidations catalyzed by human cytochrome P450 enzymes: a comparison with SKF-525A and ketoconazole. Drug Metab Pharmacokinet 18:287-295.

Eugster HP, Sengstag C, Hinnen A, Meyer UA, and Würgler FE (1991) Heterologous expression of human microsomal epoxide hydrolase in Saccharomyces cerevisiae. Study of the valpromidecarbamazepine epoxide interaction. Biochem Pharmacol 42:1367-1372.

Gill SS, Ota K, and Hammock BD (1983a) Radiometric assays for mammalian epoxide hydrolases and glutathione S-transferase. Anal Biochem 131:273-282.

Gill SS, Ota K, Ruebner B, and Hammock BD (1983b) Microsomal and cytosolic epoxide hydrolases in rhesus monkey liver, and in normal and neoplastic human liver. Life Sci 32.2693-2700.

Gill SS, Wie SI, Guenthner TM, Oesch F, and Hammock BD (1982) Rapid and sensitive enzyme-linked immunosorbent assay for the microsomal epoxide hydrolase. Carcinogenesis 3:1307-1310.

Hechtberger P, Wirnsberger G, Mischitz M, Klempier N, and Faber K (1993) Asymmetric hydrolysis of epoxides using an immobilized enzyme preparation from Rhodococcus sp. Tetrahedron Asymmetry 4:1161-1164.

Hosagrahara VP, Rettie AE, Hassett C, and Omiecinski CJ (2004) Functional analysis of human microsomal epoxide hydrolase genetic variants. Chem Biol Interact 150:149-159.

Johansson A, Löfberg C, Antonsson M, von Unge S, Hayes MA, Judkins R, Ploj K, Benthem L, Lindén D, Brodin P, et al. (2016) Discovery of (3-(4-(2-Oxa-6-azaspiro[3.3]heptan-6ylmethyl)phenoxy)azetidin-1-yl)(5-(4-methoxyphenyl)-1,3,4-oxadiazol-2-yl)methanone (AZD1979), a melanin concentrating hormone receptor 1 (MCHr1) antagonist with favorable physicochemical properties. J Med Chem 59:2497-2511.

Kerr BM, Rettie AE, Eddy AC, Loiseau P, Guyot M, Wilensky AJ, and Levy RH (1989) Inhibition of human liver microsomal epoxide hydrolase by valproate and valpromide: in vitro/in vivo correlation. Clin Pharmacol Ther 46:82-93.

Kitteringham NR, Davis C, Howard N, Pirmohamed M, and Park BK (1996) Interindividual and interspecies variation in hepatic microsomal epoxide hydrolase activity: studies with cis-stilbene oxide, carbamazepine 10, 11-epoxide and naphthalene. J Pharmacol Exp Ther 278:1018-1027.

Kroetz DL, Loiseau P, Guyot M, and Levy RH (1993) In vivo and in vitro correlation of microsomal epoxide hydrolase inhibition by progabide. Clin Pharmacol Ther 54:485-497.

Li XQ, Hayes MA, Grönberg G, Berggren K, Castagnoli N, Jr, and Weidolf L (2016) Discovery of a novel microsomal epoxide hydrolase-catalyzed hydration of a spiro oxetane. Drug Metab Dispos 44:1341-1348.

Liu JY, Park SH, Morisseau C, Hwang SH, Hammock BD, and Weiss RH (2009) Sorafenib has soluble epoxide hydrolase inhibitory activity, which contributes to its effect profile in vivo. Mol Cancer Ther 8:2193-2203.

Morisseau C, Bernay M, Escaich A, Sanborn JR, Lango J, and Hammock BD (2011) Development of fluorescent substrates for microsomal epoxide hydrolase and application to inhibition studies. Anal Biochem 414:154-162.
Morisseau C and Hammock BD (2005) Epoxide hydrolases: mechanisms, inhibitor designs, and biological roles. Annu Rev Pharmacol Toxicol 45:311-333.

Morisseau C and Hammock BD (2007) Measurement of soluble epoxide hydrolase (sEH) activity, in Curr Protoc Toxicol 4:4.23.1-4.23.18, Wiley Interscience, doi: 10.1002/0471140856 tx0423s33.

Müller F, Arand M, Frank H, Seidel A, Hinz W, Winkler L, Hänel K, Blée E, Beetham JK, Hammock BD, et al. (1997) Visualization of a covalent intermediate between microsomal epoxide hydrolase, but not cholesterol epoxide hydrolase, and their substrates. Eur J Biochem 245 : 490-496.

Oesch F, Herrero ME, Hengstler JG, Lohmann M, and Arand M (2000) Metabolic detoxification: implications for thresholds. Toxicol Pathol 28:382-387.

Pacifici GM, Franchi M, Bencini C, and Rane A (1986) Valpromide inhibits human epoxide hydrolase. Br J Clin Pharmacol 22:269-274.

Rioux N, Duncan KW, Lantz RJ, Miao X, Chan-Penebre E, Moyer MP, Munchhof MJ, Copeland RA, Chesworth R, and Waters NJ (2016) Species differences in metabolism of EPZ015666, an oxetane-containing protein arginine methyltransferase-5 (PRMT5) inhibitor. Xenobiotica 46: $268-277$.

Seidegård J and DePierre JW (1980) Benzil, a potent activator of microsomal epoxide hydrolase in vitro. Eur J Biochem 112:643-648.

Song W, Yu L, and Peng Z (2015) Targeted label-free approach for quantification of epoxide hydrolase and glutathione transferases in microsomes. Anal Biochem 478:8-13.

Steinreiber A and Faber K (2001) Microbial epoxide hydrolases for preparative biotransformations. Curr Opin Biotechnol 12:552-558.

Stepan AF, Karki K, McDonald WS, Dorff PH, Dutra JK, Dirico KJ, Won A, Subramanyam C, Efremov IV, O'Donnell CJ, et al. (2011) Metabolism-directed design of oxetane-containing arylsulfonamide derivatives as $\gamma$-secretase inhibitors. J Med Chem 54:7772-7783.

Thomas PE, Ryan DE, von Bahr C, Glaumann H, and Levin W (1982) Human liver microsoma epoxide hydrolase. Correlation of immunochemical quantitation with catalytic activity. $\mathrm{Mol}$ Pharmacol 22:190-195.

Tzeng HF, Laughlin LT, Lin S, and Armstrong RN (1996) The catalytic mechanism of microsomal epoxide hydrolase involves reversible formation and rate-limiting hydrolysis of the alkyl-enzyme intermediate. J Am Chem Soc 118:9436-9437.

Václavíková R, Hughes DJ, and Souček P (2015) Microsomal epoxide hydrolase 1 (EPHX1): gene, structure, function, and role in human disease. Gene 571:1-8.

Widersten M, Gurell A, and Lindberg D (2010) Structure-function relationships of epoxide hydrolases and their potential use in biocatalysis. Biochim Biophys Acta 1800:316-326.

Wuitschik G, Carreira EM, Wagner B, Fischer H, Parrilla I, Schuler F, Rogers-Evans M, and Müller K (2010) Oxetanes in drug discovery: structural and synthetic insights. J Med Chem 53:3227-3246.

Address correspondence to: Dr. Martin A. Hayes, Cardiovascular and Metabolic Diseases, Innovative Medicines and Early Development, AstraZeneca, Pepparedsleden 1, Mölndal, 431 83, Sweden. E-mail: martin.hayes@astrazeneca.com 